Multimodality imaging of

\section{unilateral absence of the right pulmonary artery}

A 5-month-old boy was admitted to our hospital because of cyanosis when crying. Chest X-ray revealed an obviously enlarged heart shadow (Fig. 1a). Physical examination revealed a systolic murmur at the left sternal border. Transthoracic twodimensional echocardiography showed severely dilated right atrium and right ventricle with impaired right ventricular systolic function. Left atrial and left ventricular compressions were observed (Fig. 1b). Color Doppler imaging revealed severe tricuspid regurgitation (Fig. 1c). Continuous wave Doppler echocardiography indicated significantly elevated pulmonary artery systolic pressure (103 mm Hg) (Fig. 1d). Two-dimensional and color Doppler echocardiography demonstrated a dilated left pulmonary artery, although the right pulmonary artery was not detected (Fig. 1e, 1f, Video 1). Three dimensional volume-rendered computed tomography of the chest and computed tomography angiography displayed the dilated left pulmonary artery, but the right pulmonary artery was absent (Fig. 2a, 2b). Pulmonary artery angiography confirmed the absence of right pulmonary artery and a dilated main pulmonary artery (Fig. 2c, Video 2). Aortic artery angiography revealed a collateral vessel arising from the right subclavian artery to the right lung (Fig. 2d, Video 3). Therefore, the diagnosis of unilateral absence of the right pulmonary artery (UARPA) was made on the basis of aforementioned findings of multimodality imaging.

UARPA is a rare congenital malformation with an incidence ranging from 1 in 200,000 to 1 in 300,000 (1). Early diagnosis of UARPA still remains challenging owing to a lack of typical clinical features (2). Our case emphasizes that multimodality imaging techniques play an important role in making an accurate diagnosis.

Acknowledgment: Authors would like to thank Dr. Yan Zhang for her contribution to picture preparation.

Institutional and financial support: The study was supported by National Key R\&D Program of China (Grant Nos. 2018YFC0114600), the National Natural Science Foundation of China (Grant Nos.81727805, 81530056 and 81671705) and the Key Research and Development Program of Hubei (Grant No. 2020DCD015).

Informed consent: Informed consent was obtained from the patient's guardian.
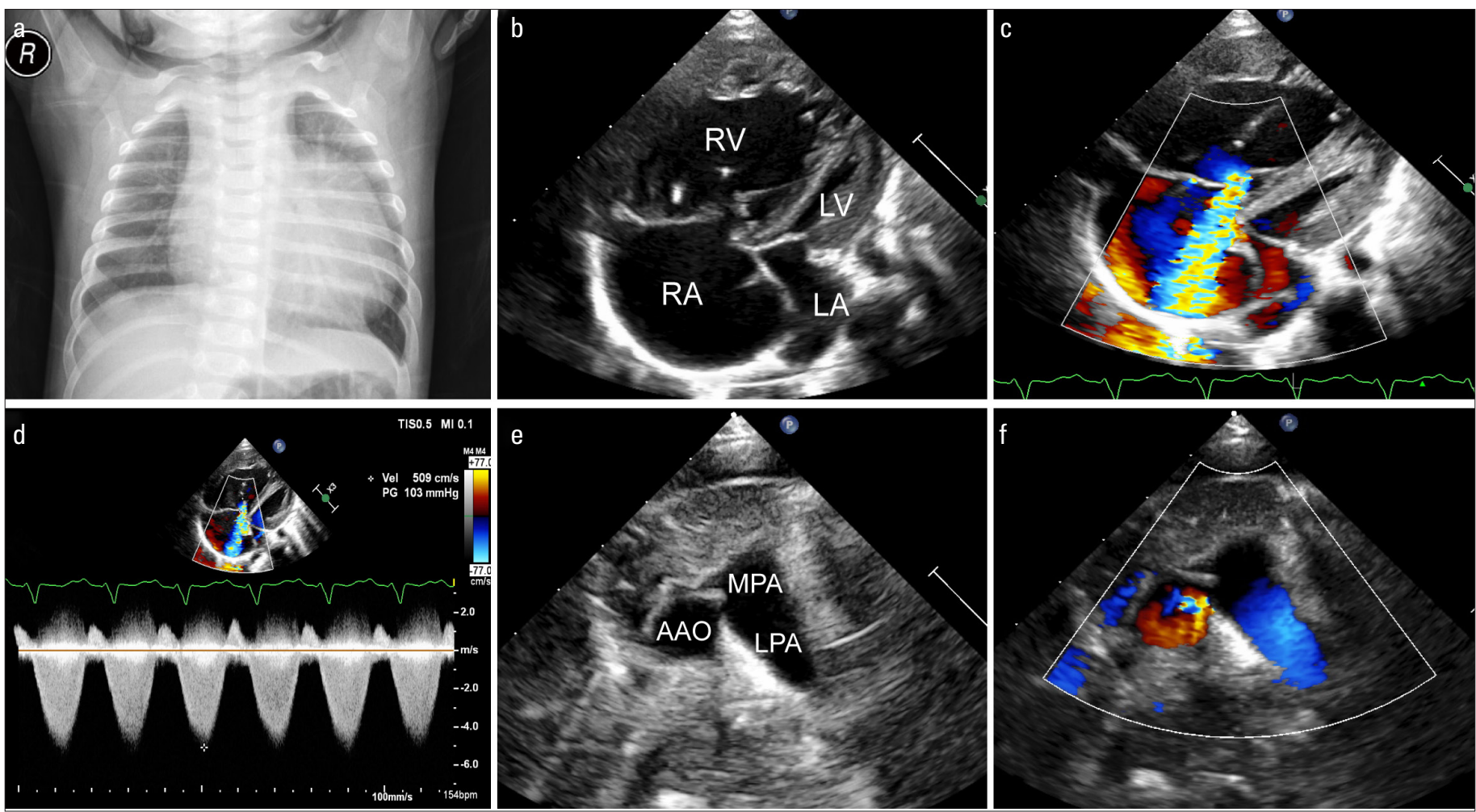

Figure 1. Chest X-ray and echocardiography images in a child with unilateral absence of the right pulmonary artery. Chest X-ray revealing an obviously enlarged heart shadow (a). Transthoracic two-dimensional echocardiography showing dilated right atrium and right ventricle, along with compressed left atrium and left ventricle (b). Color Doppler flow imaging indicating severe tricuspid regurgitation (c). Continuous-wave Doppler echocardiography exhibiting an elevated pulmonary artery systolic pressure (d). Two-dimensional and color Doppler echocardiography demonstrating the absence of right pulmonary artery with dilated left pulmonary artery $(\mathrm{e}, \mathrm{f})$.

RV - right ventricle; RA - right atrium; LV - left ventricle; LA - left atrium; MPA - main pulmonary artery; AAO - ascending aorta; LPA - left pulmonary artery. 


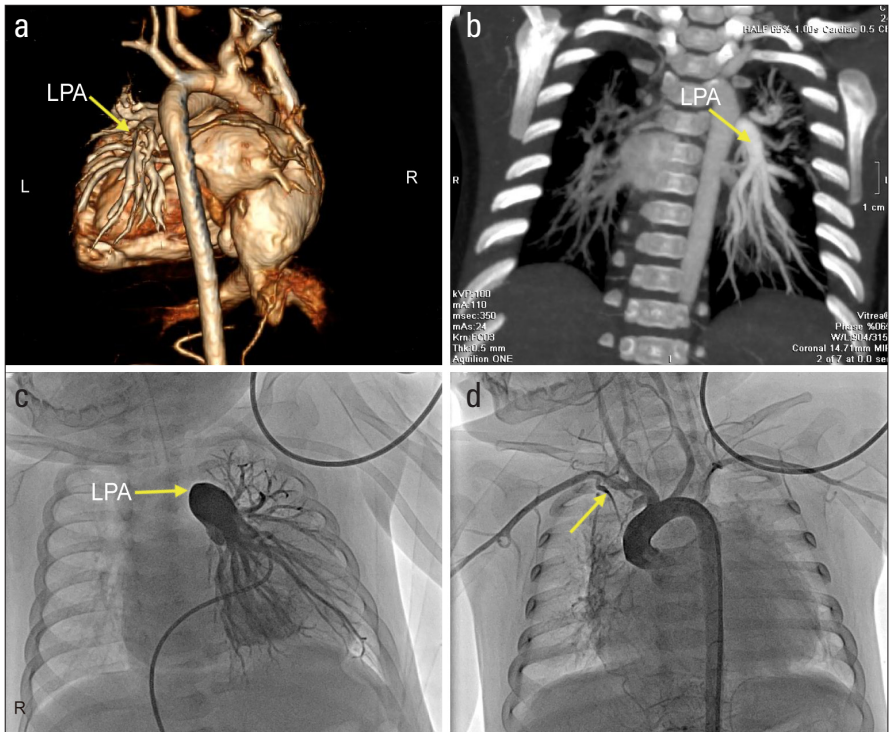

Figure 2. Chest computed tomography and Cardiovascular angiography images in a child with UARPA. Three-dimensional CT angiography and reconstruction confirming the absence of right pulmonary artery with dilated left pulmonary artery (yellow arrow, a, b). Pulmonary artery angiography exhibits that the right pulmonary artery is absent (c). Aortic artery angiography revealing a collateral vessel arising from the right subclavian artery to the right lung (yellow arrow) (d) LPA - left pulmonary artery

Video 1. Two-dimensional echocardiography demonstrating the absence of right pulmonary artery with a dilated left pulmonary artery

Video 2. Pulmonary artery angiography confirming the absence of right pulmonary artery

Video 3. Aortic artery angiography indicating a collateral vessel arising from the right subclavian artery to the right lung

\section{References}

1. Ozgun Niksarlıoğlu EY, Yeter A, Cetinkaya A, Uysal MA, Çamsarı G. An elderly patient and an adult patients with isolated unilateral pulmonary arterial hypoplasia: Two cases reports and literature review of the literature. Respir Med Case Rep 2017; 22: 106-8. [Crossref]

2. Griffin N, Mansfield L, Redmond KC, Dusmet M, Goldstraw P, Mittal TK, et al. Imaging features of isolated unilateral pulmonary artery agenesis presenting in adulthood: a review of four cases. Clin Radiol 2007; 62: 238-44. [Crossref]

\section{Oingchang Chen1,*, \# (D), Yuman Li1,*, \# (D), Zhengwu Tan** (D), Li Zhang1, * (D), Mingxing Xie 1, * (D) \\ Departments of *Ultrasound Medicine, and **Radiology, Union Hospital, Tongji Medical College, Huazhong University of Science and Technology; Wuhan-China \\ ${ }^{1}$ Hubei Province Key Laboratory of Molecular Imaging; Wuhan- China}

\#These authors contributed equally to this work.

Address for Correspondence: Mingxing Xie, MD,

Department of Ultrasound Medicine, Union Hospital, Tongji Medical College, Huazhong University of Science and Technology; 1277 Jiefang Avenue,

Wuhan-China

Phone: 86-27-85726430

E-mail: xiemx@hust.edu.cn

CCopyright 2021 by Turkish Society of Cardiology -

Available online at

www.anatoljcardiol.com

DOI:10.5152/AnatolJCardiol.2021.166 